\title{
Exploring simultaneous perturbation profiles in milk yield and body weight reveals a diversity of animal responses and new opportunities to identify resilience proxies
}

\author{
A. Ben Abdelkrim, ${ }^{1,2 *} \odot$ T. Tribout, ${ }^{1}$ O. Martin, ${ }^{2}$ D. Boichard, ${ }^{1}$ (ㄴ) V. Ducrocq, ${ }^{1}$ and N. C. Friggens ${ }^{2}$ \\ ${ }^{1}$ Université Paris-Saclay, INRAE, AgroParisTech, UMR GABI, 78350 Jouy-en-Josas, France \\ ${ }^{2}$ Université Paris-Saclay, INRAE, AgroParisTech, UMR MoSAR, 75005 Paris, France
}

\section{ABSTRACT}

Livestock husbandry aims to manage the environment in which animals are reared to enable them to express their production potential. However, animals are often confronted with perturbations that affect their performance. Evaluating effects of these perturbations on animal performance could provide metrics to quantify and understand how animals cope with their environment, and therefore to better manage them. Body weight (BW) and milk yield (MY) dynamics over lactation may be used for this purpose. The goal of this study was to estimate an unperturbed performance trajectory using a differential smoothing approach on both MY and BW time series, and then to identify the perturbations and extract their phenotypic features. Daily MY and BW records from 490 primiparous Holstein cows from 33 commercial French herds were used. From the fitting procedure, estimated unperturbed performance trajectories of BW and MY were clustered into 3 groups. After the fitting procedure, 1,754 deviations were detected in the MY time series and 964 were detected in the BW time series across all cows. Overall, 425 of these deviations were detected during the same period $( \pm 10 \mathrm{~d})$ in both MY and BW time series, 76 of which started at the same time. Results suggest that combining various individual dynamic measures and revealing the relationship that exists between them could be of great value in obtaining reliable estimates of resilience components in large populations.

Key words: milk yield, body weight, resilience, perturbation, clustering smoothing

Received March 16, 2020.

Accepted August 27, 2020.

*Corresponding author: ahmed.ben-abdelkrim@inra.fr

\section{INTRODUCTION}

In livestock production, real-time monitoring technologies are becoming widely available. These technologies have become an increasingly important element in allowing us to maximize the profitability of dairy farms by identifying the most efficient animals (e.g., animals that produce a given quantity of milk using less resources), thereby contributing to the reduction of their ecological footprint and ensuring the environmental and economic sustainability of dairy production (Lebacq et al., 2015). An increasing number of dairy farms use automatic milking systems that are often equipped with technologies for automatically recording both milk production and $\mathrm{BW}$ of the animal on a regular basis. This provides useful time-series data concerning 2 of the key phenotypes involved in animal efficiency. However, the raw data are meaningless and must be transformed into information that can be interpreted for use by decision-making tools. The challenge is in capturing this information and developing the appropriate tools to analyze it.

High-frequency BW and milk yield (MY) measurements play a useful role in the assessment of the animals' value and provide information of previously unreachable resolution. Such measurements can give an improved estimate of the achievable curve of animal performance but can also be used to detect deviations from this potential curve (Codrea et al., 2011). These deviations can have several causes, including physical (e.g., disease, climate, feeding), psychological (e.g., novel environment, social stressor, human interaction), or both physical and psychological causes, and the same stress does not necessarily have the same effect on different animals. Methods able to quantify the gap between potential and perturbed trajectories can give an indirect quantification of the animal's resilience. Berghof et al. (2019a), based on the work of Colditz and Hine (2016), define resilience as the capacity of the animal to be minimally affected by disturbances or to 
rapidly return to the state pertained before exposure. Over the last decade, several authors have developed models that provide the necessary elements to be able to characterize resilience. Some authors have proposed models to compare the variation of performance during short- and long-term periods (e.g., total BW time series, lactation or part of lactation), thereby quantifying the value of resilience and adaptive capacity. Puillet and Martin (2017) developed a compartmental model to convert the BW trajectory of an animal into vectors of biologically meaningful parameters (i.e., growth, body reserves balance, uterine load, and maternal investment components). The vectors representing the underlying mechanisms of growth may provide new metrics to help our understanding of complex phenotypes such as resilience. Ben Abdelkrim et al. (2020) developed a perturbed lactation model that defines a theoretical unperturbed lactation curve and calculates actual deviations from this unperturbed lactation model. The same approach was proposed for BW by Martin and Ben Abdelkrim (2019). These 2 models offer a way of quantifying the consequences of external natural factors and exploring hypotheses about the biological types of responses caused by specific perturbations. To explore the variation between individuals in the response to and recovery from a nutritional challenge and the repeatability of responses between lactation stages was an approach explored by Friggens et al. (2016) as a means to quantify differences in resilience profiles. These types of models offer insight on the diversity of the perturbations and show that they may affect different biological traits.

The response of an animal to stressors is a dynamic phenomenon that results in a deviation from unperturbed performance. These deviations are defined by several parameters, which are directly linked to the phases of collapse and recovery of animal performance (Friggens et al., 2016). Several studies have defined resilience as the capacity of an animal to minimize the effect of perturbations and maintain homogeneity of productivity (Berghof et al., 2019a; Adriaens et al., 2020; Poppe et al., 2020). The main objective of this study was to propose tools that provide metrics to assess deviations in animal performance and, as a consequence, propose candidate proxies of resilience. The strategy for reaching this objective was (1) to propose an approach to estimate unperturbed performance trajectories for MY and for BW based on actual observations; (2) to explore the relationships between MY and BW unperturbed profiles based on this estimation; (3) to develop a method to detect the perturbations present in performance trajectories; and (4) to analyze the variability in perturbations, extract quantifiable features of the perturbation profiles, and explore relationships between
$\mathrm{MY}$ and BW in response to perturbations that affect performance under commercial on-farm conditions.

\section{MATERIALS AND METHODS}

\section{Data}

The data used in the present study were recorded on Holstein cows in 33 commercial dairy farms located in western France. These herds were members of Bretagne Conseil Elevage Ouest (BCEL-Ouest). The farms were equipped with milking robots with automatic weighing platforms and a closed waiting area in front of the unit entrance. These robots recorded the date and time of the visit, the weight of collected milk, and BW of the cow. The milk records were standardized into a 24 -h milk yield according to the equation $M Y=\sum_{\text {day }} M Y_{R} \times \frac{1,440}{\Delta_{\text {time }}}$, where $M Y_{R}$ is the weight of collected milk recorded at each visit of the cow in the milking unit, and $\Delta_{\text {time }}$ is the time interval (in minutes) between the last milking of the current test-day and the last milking of the previous test-day. The daily mean of the recorded BW was calculated. The data set contained 108,444 records for milk and 97,741 records for BW from 490 first-lactation cows calving between February 2013 and May 2017. To reduce the effect of missing data on the performance curves' shape and assess the distribution of perturbation responses between different lactation stages, lactations with fewer than 150 records or with more than 35\% missing data for MY or BW were discarded. The whole lactation was considered, up to 717 DIM.

\section{Smoothing Method}

All data processing and statistical analyses were performed using R (https://www.r-project.org/). The smoothing approach described by Ramsay and Silverman (2005) was used (package "fda": Ramsay et al., 2018). The base function, denoted $\left[f_{\text {perf }}(\mathbf{c})\right]$, being minimized is

$$
f_{\text {perf }}(\mathbf{c})=\sum_{i=1}^{n}\left[y_{i}-x\left(t_{i}\right)\right]^{2}+\lambda \int_{0}^{L}\left[\partial^{4} x(\mathbf{c}, \mathbf{t})\right]^{2} \mathrm{~d} t
$$

with

$$
x(\mathbf{c}, \mathbf{t})=\mathbf{c}^{\prime} \varphi(\mathbf{t})
$$

where $y_{i}$ represent $n$ observed values of $\mathrm{MY}$ or $\mathrm{BW}$ in the time-series between $\mathrm{d} 0$ and $L ; x(\mathbf{c}, \mathbf{t})$ is the smooth 
function built combining $\varphi(\mathbf{t})$ basis B-spline functions, which are linearly combined with coefficients vector $\mathbf{c}$. The notation $\partial^{4} x(\mathbf{c}, \mathbf{t})$ refers to the fourth derivative of $x(\mathbf{c}, \mathbf{t})$ with respect to time $(t)$ and defined by its differential $(d t)$. The $\lambda$ parameter controls the relative emphasis given to goodness of fit versus smoothness. When $\lambda$ is low, $x(\mathbf{c}, \mathbf{t})$ tends to adjust the data as well as possible by reducing the squared error. The larger the value of $\lambda$, the more weight is put on the penalty term and thus the second derivative evolves toward to a straight line. Therefore, differential smoothing of time-series measurements consists of changing the value of $\lambda$. Regarding a priori assumptions constraining the shape of the lactation curve or the distance between spline knots and the different values of $\lambda$, we relied on the study of Codrea et al. (2011). Briefly, those authors used 2 values of $\lambda$ : one was relatively small value $(\lambda=$ $2,500)$ to capture the short-term variations (STV), and the other was larger $\left(\lambda=10^{6}\right)$ and approximated the longer-term phenotypic potential performance (LTV) of the milk yield curve. The difference between LTV and STV provides different measures of perturbations. In general, LTV curves are characterized by a high $\lambda$ value, reflecting the inherent shape of the cow performance curves without perturbations. However, it should be noted that long-term influences on milk yield (e.g., technical problems in the automatic milking system or data of the animals that have sustained a large constraint) can in extremis influence the general shape of these curves. For the LTV curves, a quantile regression was used to minimize the effect of data coming from periods of perturbation on the estimation of the LTV curves; that is, to better approach the undisturbed phenotypic potential of each cow. For this, we based our work on that of Codrea et al. (2011), who proposed a method for deriving the undisturbed phenotypic potential using quantile regression with confidence limits of 90 and $60 \%$. With this approach, named "quantile envelope," the LTV curve was obtained by smoothing performance of data points present between the $60 \%$ and $90 \%$ quantiles and using a larger value of $\lambda$ (Figure 1). The purpose of using fairly high quantile limits was to eliminate the effect of perturbations that tend to lower the general shape of the lactation curve. The same procedure was applied to the BW data set, but taking into account the biological difference between the 2 types of measures. Indeed, BW data generally include noise mainly due to the variation in gut fill associated with the temporal pattern of meals ingested by the cow. This noise could be eliminated by quantile regression using the $20 \%$ lower quantile of the distribution in a cubic spline function, in accordance with
Thorup et al. (2012). Therefore, 2 additional modifications were necessary. The first modification concerned the limits of the quantile regression; only data points within the $10 \%$ and $30 \%$ quantiles were considered for BW (Figure 1). Furthermore, to capture the weight decline in early lactation, we found it necessary to use nonequidistant spline knots. Knots at 1, 12, 20, 60, 115, and 150 DIM were chosen, as in Friggens et al. (2007).

\section{Classification of Cow Performance}

After determination of the LTV curves of the undisturbed phenotypic potential of BW and MY, an unsupervised classification was performed using the $k$-means clustering algorithm to group the time-series segments. This clustering did not consider any fixed factors such as herd, parity, or breed. As all cows were primiparous Holsteins, the factors breed and parity were not relevant. We chose not to adjust for herd because this allows more global inferences to be drawn from any relationships between performance clusters and perturbation characteristics. To achieve this, the R package "kml" (Genolini et al., 2015) was used. This package is an implementation of $k$-means designed to be applied specifically on longitudinal data. We reiterate that $k$-means is a hill-climbing algorithm (Everitt et al., 2001) belonging to the expectation-maximization (EM; Celeux and Govaert, 1992) class. The analysis was carried out in 2 stages: each time-series segment was assigned to a cluster and then optimal clustering was reached by alternating the calculation of the centers of each cluster and assigning each observation to its "nearest cluster" by minimizing the within-cluster sum of squares. To choose the optimal number of clusters, we used the Caliński and Harabasz criterion (Caliński and Harabasz, 1974).

\section{Identification of Deviation Profiles}

The next step was to develop a method to identify the deviations from the undisturbed phenotypic potential trajectories in the MY and BW data set. The methodology used, called "residual filtered," consists for the LTV in making a first smoothing with $\lambda=$ $10^{6}$, then identifying extreme values and, to minimize their effect on the production curve, replacing all those corresponding to residuals larger than 1 standard deviation (SD) by this bound. Then, a second smoothing was performed on this reduced data set using the same $\lambda$ to obtain the final LTV. For construction of the STV curve using the full data set, a small value of $\lambda(\lambda$ $=2,500)$ was selected. The approach used to identify the start of a deviation was to find the intersection 

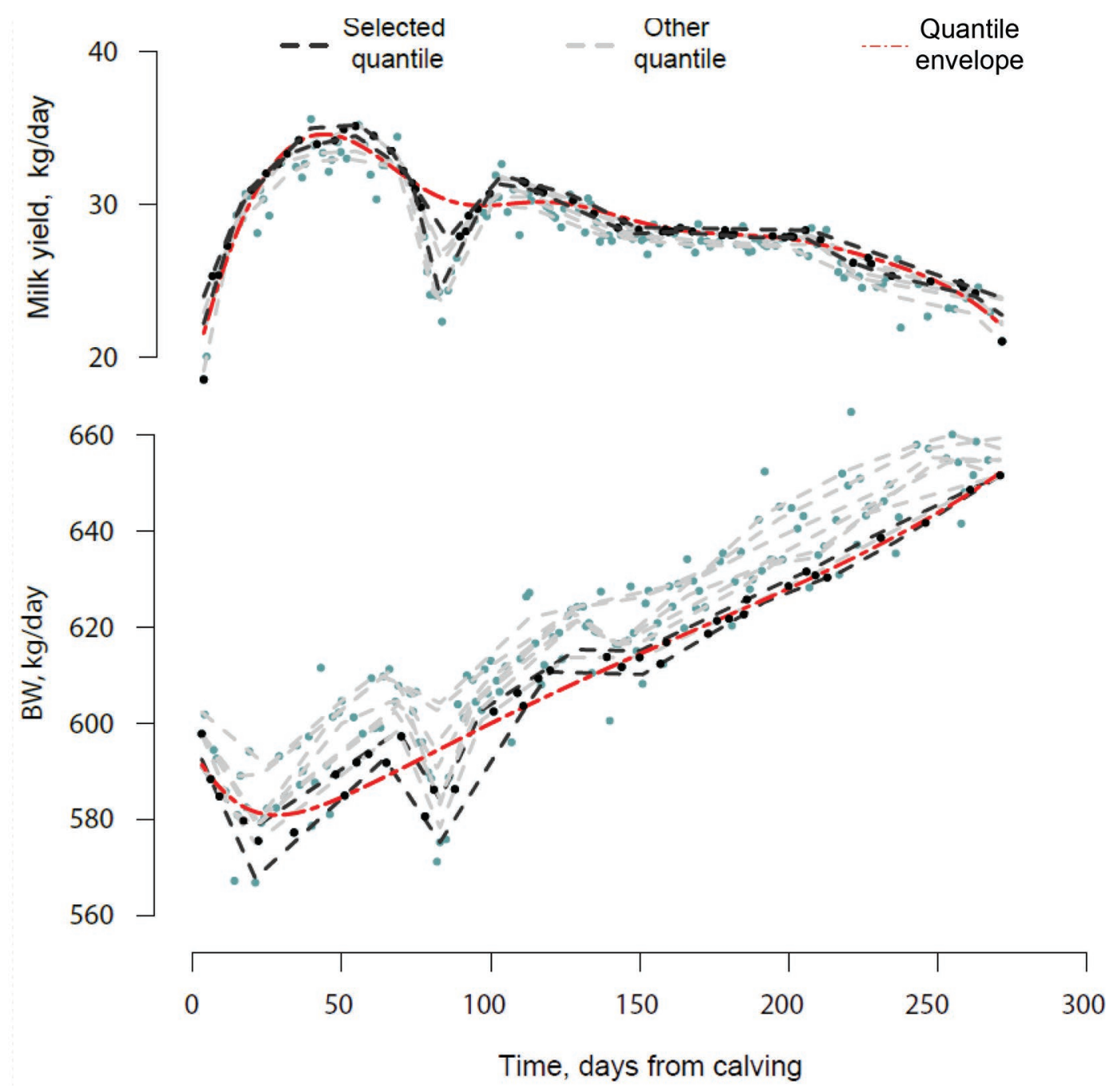

Figure 1. Construction of undisturbed phenotypic potential curve by the quantile envelope approach using the quantile regression to offset bias in the performance records. The resulting curve obtained by smoothing (smoothing parameter $\lambda=10^{6}$ ) using data points within the $60 \%$ and $90 \%$ quantiles for milk yield and the $10 \%$ and $30 \%$ quantiles for BW (black points). Data points outside these ranges (green points) are not accounted for during construction of undisturbed phenotypic potential curve.

between the STV and LTV curves. An additional step was needed to focus on the most informative deviations. This step consisted of keeping the deviations that were negative relative to the potential curve and selecting those that have a downward collapse phase and upward recovery phase (which can be regarded as key characteristics of perturbations). Deviations that did not show a downward collapse phase were discarded in this study. To eliminate trivially small deviations, we kept only deviations for which the difference between the areas under the curve of the STV and LTV curve divided over the duration of the deviation was greater than $0.5 \mathrm{~kg}$ for MY and $5 \mathrm{~kg}$ for BW. A second filter was applied to eliminate deviations without a phase of collapse. The next step was to determine perturbations that were concurrent for both MY and BW. We considered that a perturbation was concurrent if it was detected for both MY and BW within a period of $10 \mathrm{~d}$.

\section{Characterization and Classification of Concurrent Perturbation Profiles}

The concurrent perturbations were targeted to determine the existing different phenotypes. The first step of the process was to select variables that define a perturbation; the following variables were used: the area between the STV curve and the LTV curve (area under the curve, AUC, considered here as the estimated performance loss during the whole period of the perturbation), the duration of the perturbation $(\Delta \mathrm{T})$, the duration of the collapse $\left(\mathrm{T}_{\text {col }}\right)$ and recovery $\left(\mathrm{T}_{\text {rec }}\right)$ phases, the maximum value of the difference between 
Table 1. Abbreviations and variables selected to define a perturbation

\begin{tabular}{ll}
\hline Symbol & Definition \\
\hline STV & Short-term variations curve \\
LTV & Long-term phenotypic potential performance curve \\
AUC & Areas under the curve of the difference between the STV and LTV curves \\
$A U C_{L D}$ & Estimation of loss per day (AUC $/ \Delta \mathrm{T})$ \\
$\Delta \mathrm{T}$ & Duration of deviation detected \\
$\mathrm{T}_{\text {col }}$ & Collapse phase time by deviation \\
$\mathrm{T}_{\text {rec }}$ & Recovery phase time by deviation \\
$\mathrm{M}_{\mathrm{ML}}$ & Maximum performance loss during deviation \\
$\mathrm{V}_{\min }$ & Minimum value recorded during the deviation \\
\hline
\end{tabular}

LTV and STV recorded during the deviation $\left(\mathrm{M}_{\mathrm{ML}}\right)$, and the minimum value of the animal performance recorded during the deviation $\left(\mathrm{V}_{\min }\right.$; Table 1$)$. A logarithmic transformation was applied to the data to reduce the difference in scale between these different variables, and a principal component analysis (PCA) was carried out. The objective was to identify clusters of perturbations that can be identified using the above-mentioned variables.

For shape-based clustering of perturbation and as a complementary clustering method to confirm PCA results, we used a hierarchical agglomerative technique using Ward's linkage procedure (Ward, 1963). Ward's method is a hierarchical procedure that iteratively merges groups of perturbations represented by points in a Euclidean space resulting in the smallest increase in the sum of within-group sums of squares. Among agglomerative clustering methods, it is the only one that is based on a classical sum of squares criterion, producing groups that minimize within-group dispersion at each binary fusion. This method tends to divide dense clusters in an acceptable manner (Sneath and Sokal, 1973) and allowed us to highlight the different perturbation profiles.

\section{RESULTS}

We first present the clustering of phenotypic potential trajectories (LTV) and then present results pertaining to the short-term deviations (STV).

\section{Undisturbed Phenotypic Potential Curve}

To estimate the undisturbed phenotypic potential curve, the quantile envelope approach was applied. This approach is robust to different residual variations that may exist at different specific times after calving. Figure 2 shows an example of the results of the potential phenotypic curve of MY and BW obtained after smoothing.

The clustering analysis carried out on the potential phenotypic milk yield curves revealed 3 groups, essen- tially distinguished by their level of production. These groups contained $31.8,45.5$, and $22.7 \%$ of cows in higher $\left(\mathbf{H}_{\mathrm{MY}}\right)$, medium $\left(\mathbf{M}_{\mathrm{MY}}\right)$, and lower $\left(\mathbf{L}_{\mathrm{MY}}\right)$ production groups, respectively (Figure 3a).

The average milk production per day of the unperturbed lactation curve were $32.71,27.49$, and 22.52 $\mathrm{kg} / \mathrm{d}$, with peaks of $37.44,32.53$, and $27.55 \mathrm{~kg}$ for the $\mathrm{H}_{\mathrm{MY}}, \mathrm{M}_{\mathrm{MY}}$, and $\mathrm{L}_{\mathrm{MY}}$ groups, respectively. These peak values were detected at d 52 for $\mathrm{H}_{\mathrm{MY}}$, d 49 for $\mathrm{M}_{\mathrm{MY}}$, and $\mathrm{d} 44$ for $\mathrm{L}_{\mathrm{MY}}$ from calving. Three groups were also identified for the phenotypic potential BW curves. The largest group ( $\left.\mathbf{M}_{\mathrm{BW}}\right)$ included $49.8 \%$ of the cows, with an average $\mathrm{BW}$ of $588 \mathrm{~kg}$. The second group by number of cows $\left(\mathbf{L}_{\mathrm{BW}}\right)$ included $35.3 \%$ of the cows, with an average BW of $531 \mathrm{~kg}$. The last group $\left(\mathbf{H}_{\mathrm{BW}}\right)$ contained only $14.9 \%$ of cows, with an average BW of $607 \mathrm{~kg}$ (Figure $3 \mathrm{~b}$ ). The minimal value of $\mathrm{BW}$ was detected for the $\mathrm{H}_{\mathrm{MY}}, \mathrm{M}_{\mathrm{MY}}$, and $\mathrm{L}_{\mathrm{MY}}$ groups at around 21, 23, and $24 \mathrm{~d}$ after calving, respectively. The relationship between these milk and BW profile classes is shown in Table 2. Overall, $45.3 \%$ of cows were assigned to the same production group level for both traits: $7.55 \%$ of all cows were detected in the $\mathrm{H}_{\mathrm{BW}}$ and $\mathrm{H}_{\mathrm{MY}}$ clusters, $25.10 \%$ of all cows were detected in both the $\mathrm{M}_{\mathrm{BW}}$ and $\mathrm{M}_{\mathrm{MY}}$ clusters, and $12.65 \%$ of all cows were detected in both the $\mathrm{L}_{\mathrm{BW}}$ and $\mathrm{L}_{\mathrm{MY}}$ clusters. Overall, $54.7 \%$ of cows were assigned to groups with a different level of performance. Of these, $45.1 \%$ of cows were detected in the $\mathrm{H}$ or $\mathrm{L}$ groups for one performance and group $\mathrm{M}$ for the other performance (i.e., $5.71 \%$ of $\mathrm{H}_{\mathrm{MY}}$ and $14.69 \%$ $\mathrm{L}_{\mathrm{MY}}$ were present in the $\mathrm{M}_{\mathrm{BW}}$ group, and $16.33 \%$ and $8.37 \%$ were present in the $\mathrm{M}_{\mathrm{MY}}$ group) and $9.6 \%$ were affected in either $\mathrm{H}_{\mathrm{BW}}$ and $\mathrm{L}_{\mathrm{MY}}(7.96 \%)$ or $\mathrm{H}_{\mathrm{MY}}$ and $\mathrm{L}_{\mathrm{BW}}(1.63 \%)$ (Table2).

\section{Identification of Deviations}

Deviations were identified based on the intersections between the STV and LTV curves, using the "residual filtered" method. The STV curve was built with $\lambda=$ 2,500 , which makes it possible to capture the most significant variations present in the performance trajec- 
tories. Using this approach and after removal of trivial deviations, 1,754 deviations in MY and 964 deviations in BW were detected. For MY, the cumulative loss for each deviation (i.e., the area between the LTV and STV curves) was on average $37.51 \mathrm{~kg}$. These deviations last on average $32 \mathrm{~d}$ with collapse and recovery times, on average, of $16 \mathrm{~d}$. For BW, the average of the differences between the area under the LTV and STV curves was $307 \mathrm{~kg}$ with a longer duration than MY (36 d) and collapse and recovery times of $18 \mathrm{~d}$ (Table 3 ).

\section{Concurrent Perturbations Between MY and BW}

The total number of deviations detected for both MY and BW within a period of $10 \mathrm{~d}$ was 425 (i.e., $24.2 \%$ of the deviations detected for MY and $44.1 \%$ of the deviations recorded for BW). The 1,329 deviations in MY and 539 deviations in BW removed may be considered to be perturbations affecting one specific performance and not the other (Figure 4A). Out of these 425 deviations, 76 started on the same day (i.e., $17.9 \%$ of total deviations), 47 finished on the same day (i.e., 11.1\% of total deviations), and 86 reached the minimum value on the same day (i.e., $20.2 \%$ of total deviations). It is also important to note that among the total deviations, 5 deviations (i.e., $1.2 \%$ of total deviations) started, finished, and reached the minimum level of performance at exactly the same time. Eighteen deviations had the same duration of collapse phases (started and reached the minimum level at the same time). Fourteen devia-
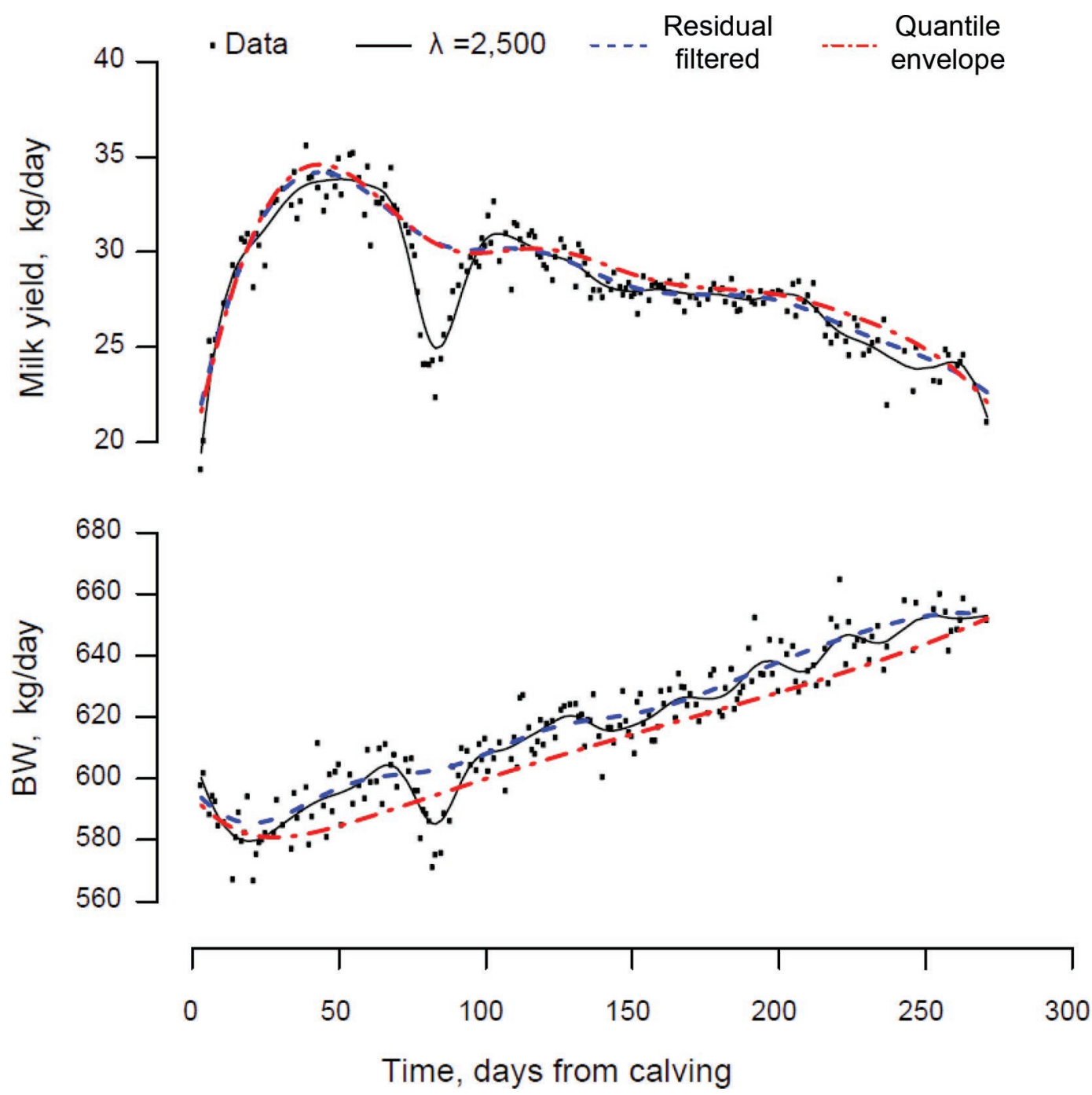

Figure 2. Different degrees of smoothing applied to lactation (milk yield) and BW curves. The black curves were built using a small value of the smoothing parameter $\lambda(\lambda=2,500)$ to capture short-term variations. The blue curves were built using the "residual filtered" method to offset bias in the yield records. The red curves were built using the "quantile envelope" method, using data points within the $60 \%$ and $90 \%$ quantiles to offset bias in the yield records. 
Table 2. Relationship and distribution percentage of individuals between the clusters obtained based on milk yield and BW

\begin{tabular}{lrcrc}
\hline \multicolumn{4}{c}{ Milk yield } \\
\cline { 2 - 5 } BW & Higher & Medium & Lower & All \\
\hline Higher & 7.5 & 16.3 & 8.0 & 31.8 \\
Medium & 5.7 & 25.1 & 14.7 & 45.5 \\
Lower & 1.6 & 8.4 & 12.6 & 22.6 \\
All & 14.9 & 49.8 & 35.3 & 100 \\
\hline
\end{tabular}

tions had the same duration of recovery phases (started and reached the minimum level at the same time) and 2 deviations started and finished at the same time, but had different collapse and recovery phases (Figure 4B).

\section{Characterization and Classification of Concurrent Perturbation Profiles}

A PCA was carried out to characterize concurrent perturbations profiles. This analysis showed that $64.2 \%$ of the total variation was explained by the first 3 principal components. The first principal component (axis 1), which explained $26.8 \%$ of the total variation, had a negative loading for $\mathrm{V}_{\text {min }}$, which was opposed to the other variables. The second principal component (axis 2 ), which explained $22.5 \%$ of the total variation, clearly separated the "time" variables $\left(\Delta \mathrm{T}, \mathrm{T}_{\text {col }}\right.$, and $\mathrm{T}_{\text {rec }}$ from the deviations) on the positive side, from the "quantity" variables (except AUC_BW) on the negative side. The dispersion of the perturbation profiles on the bi-plot space of the PCA did not reveal distinct groups (Figure 5a). This suggests that there was a continuum in the shape characteristics of the perturbation profiles. To visualize this diversity gradient, we constructed a dendrogram of classification of concurrent perturbation profiles. The dendrogram (Figure 5b) shows the pres-
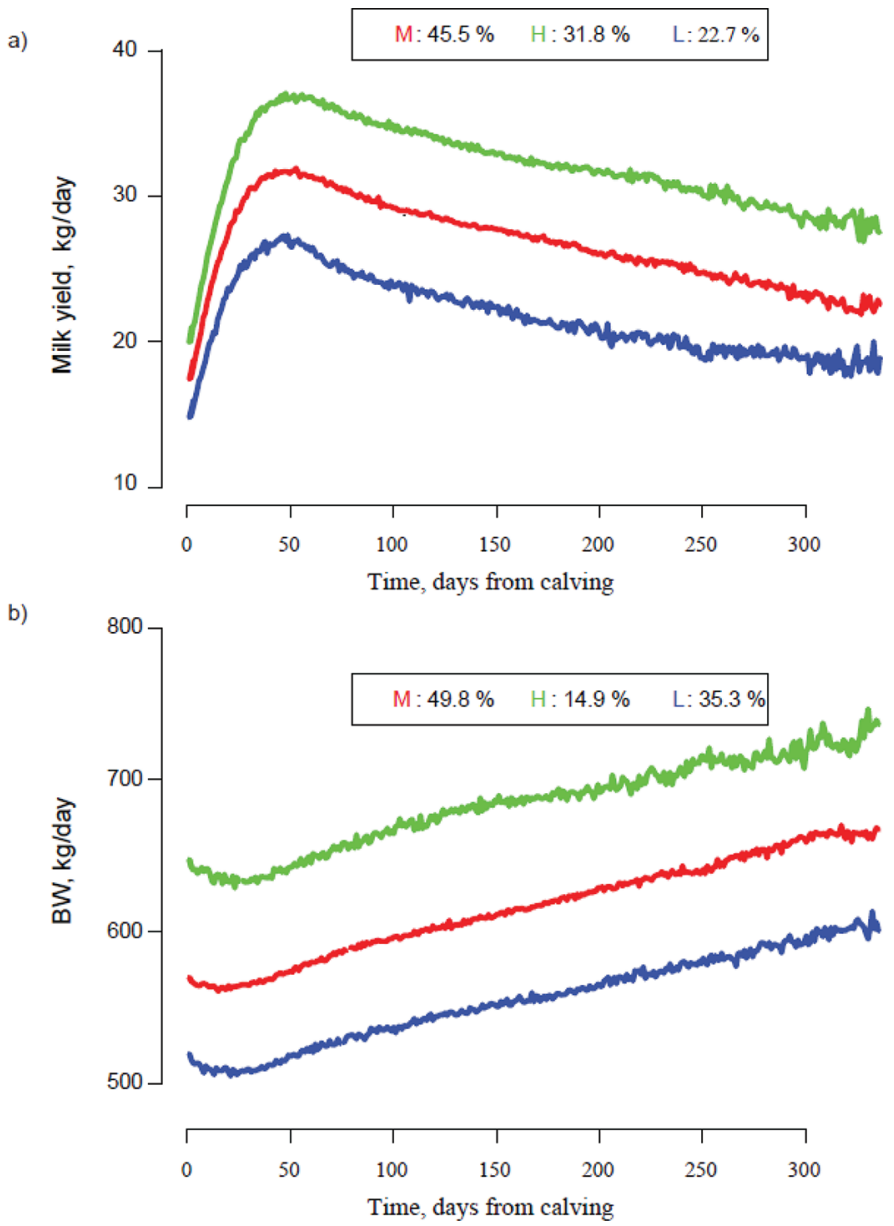

Figure 3. Curve clusters for the undisturbed phenotypic potential for (a) milk yield, and (b) BW of primiparous Holstein cows. The $\mathrm{H}$, $\mathrm{M}$, and $\mathrm{L}$ curves represent the cows in the higher, medium, and lower production and BW groups, respectively.

Table 3. Description of the deviations (range; SD given in parentheses) obtained by intersections between short-term variations (STV) and long-term components of the performance curve (LTV)

\begin{tabular}{lcc}
\hline Variable $^{1}$ & \multicolumn{1}{c}{ Milk yield } & BW \\
\hline $\mathrm{NDD}_{\mathrm{T}}$ & 1,754 & 964 \\
$\mathrm{NDD}_{\mathrm{I}}$ & $3.61(0-8 ; 1.46)$ & $3.57(0-8 ; 1.45)$ \\
$\mathrm{L}_{\text {lac }}(\%)$ & $2.33(0.88-6.97 ; 1.05)$ & $0.69(0.34-2.88 ; 0.29)$ \\
$\mathrm{L}_{\text {pert }}(\%)$ & $0.55(0.02-5.74 ; 0.56)$ & $0.20(0.01-1.71 ; 0.19)$ \\
$\mathrm{M}_{\mathrm{AUC}}(\mathrm{kg})$ & $37.51(1.55-470.47 ; 36.05)$ & $169.16(0.005-2,484.65 ; 201.84)$ \\
$\mathrm{M}_{\Delta \mathrm{T}}(\mathrm{d})$ & $32.31(3-143 ; 17.41)$ & $36(3-211 ; 22.67)$ \\
$\mathrm{M}_{\text {Tcol }}(\mathrm{d})$ & $15.99(1-141 ; 11.95)$ & $18.01(1-115 ; 14.23)$ \\
$\mathrm{M}_{\text {Trec }}(\mathrm{d})$ & $16.32(0-100 ; 11.87)$ & $17.99(0-134 ; 14.54)$ \\
$\mathrm{M}_{\mathrm{ML}}(\mathrm{kg})$ & $2.12(0.63-19.02 ; 1.52)$ & $15.36(7.25-69.90 ; 7.66)$ \\
\hline
\end{tabular}

${ }^{1} \mathrm{NDD}_{\mathrm{T}}=$ total number of deviations detected; $\mathrm{NDD}_{\mathrm{I}}=$ average of the total number of deviations detected per cow; $\mathrm{L}_{\text {lac }}(\%)=$ estimation of the total performance loss during lactation; $\mathrm{L}_{\text {pert }}(\%)=$ estimation of the performance loss during a perturbation; $\mathrm{M}_{\mathrm{AUC}}=$ average of area under the curve of the difference between the STV and LTV curves; $\mathrm{M}_{\Delta \mathrm{T}}=$ average duration of deviation detected $(\Delta \mathrm{T}) ; \mathrm{M}_{\mathrm{Tcol}}=$ average of collapse phase time by deviation $\left(\mathrm{T}_{\text {col }}\right) ; \mathrm{M}_{\mathrm{Trec}}=$ average of recovery phase time by deviation $\left(\mathrm{T}_{\mathrm{rec}}\right) ; \mathrm{M}_{\mathrm{ML}}=$ average of maximum performance loss during deviation (ML). 
ence of 2 major groups and 1 minor group. The minor group (group 1) was mainly composed of perturbations without recovery. Group 2 consisted of 191 perturbations characterized by longer durations (for MY and BW), which can result in fairly substantial performance losses. Group 3 was composed of 215 perturbations, shorter for MY than for BW. Therefore, this group showed a more reduced loss in MY than in BW (Table 4). Figure $5 \mathrm{c}$ gives some examples of perturbation phenotypes, representative of each cluster. Weak positive and negative correlations were found between the time variables and the quantity variables (except AUC_MY and AUC_BW; Figure 6).

\section{DISCUSSION}

How to extract biologically meaningful features from time-series and to quantify perturbations, whether on BW or MY, has been addressed in several recent studies (Nguyen Ba et al., 2019; Revilla et al., 2019; Ben Abdelkrim et al., 2020). The general goal of these studies was to extract metrics that are capable

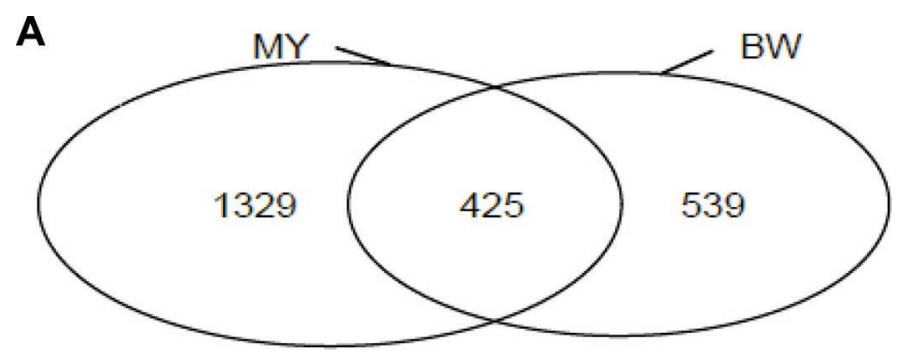

B

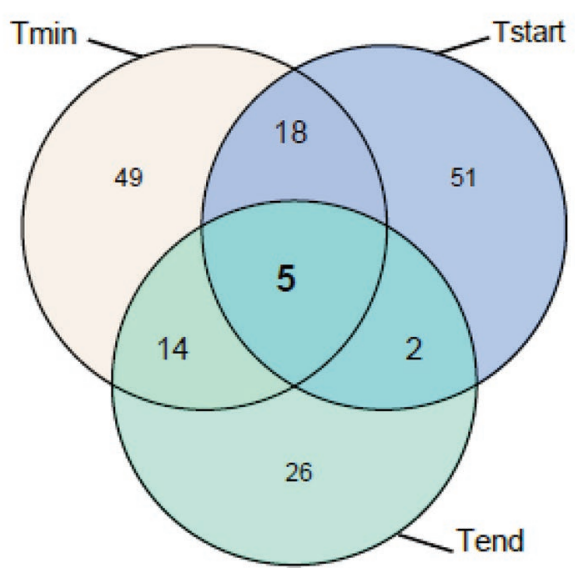

Figure 4. Venn diagram representing the number of perturbations (A) detected from the intersections between the short-term variation (STV) and long-term phenotypic potential performance (LTV) curves, in the production (milk yield, MY) and live weight (BW) trajectories, highlighting the disturbances detected during the same period; and (B) detected during the same period and starting at the same time $\left(\mathrm{T}_{\text {start }}\right)$, ending at the same time $\left(\mathrm{T}_{\text {end }}\right)$, or that reached minimum performance at the same time $\left(\mathrm{T}_{\min }\right)$. of (1) characterizing perturbations, (2) quantifying theoretical losses of production, and (3) comparing the capabilities of animals to respond to perturbations to quantify and evaluate their resilience. In these studies, several parameters arising from perturbation profiles were defined. They provide information to characterize the ability of animals to minimize or not the effects of environmental perturbations on their performance and, therefore, they provide proxies for assessing their resilience. However, these studies considered only BW or MY measurements separately, not both at the same time. Clearly, drawing general conclusions on animal resilience from responses for only one type of measure is insufficient. Animal resilience is made up of several complex elements that depend on its reaction to different origins of disturbances (physical or psychological) and its adaptation to its environment. This reaction to disturbances can differ from one animal to another, it can differ for the same animal if it occurs at different stages of its productive life, and it can also be expressed differently depending on performance. Having tools to build an overall picture of an animal's reaction to a disturbance will contribute to further refining our understanding of the complex phenomenon of resilience. The current study proposes such a tool that simultaneously tracks perturbations for more than one biological measure. This may allow the detection of animals that deal with perturbations differently; for example, that could preferentially favor a response on MY or on BW. Comparing the kinetics and dynamics of perturbations on different aspects of animal performance and measuring the degree of synchronization between responses to a particular condition may allow 2 types of inference: (1) to characterize, visualize, and compare the effect of a given type of disturbance on performance, in terms of both average response-recovery dynamics and also the individual variation in such traits; and (2) to compare animal strategies when facing different types of perturbations. Both of these aspects will be very informative for animal resilience.

The strategy we chose for the detection of perturbations had to be applicable to both types of traits, in our case by setting the same value to the smoothing parameters for BW or MY. This makes it easy to apply to large volumes of on-farm data from animals under commercial conditions. In this study, we focused on negative deviations resulting from the intersection between STV and LTV curves constructed based on the "residual filtered" method. However, in principle, the approach could also detect positive responses to perturbations. This does not seem meaningful for measures such as MY and BW but would be valuable for other indicators; for example, disease indicators such as SCC that increase during a mastitis event. 
a)

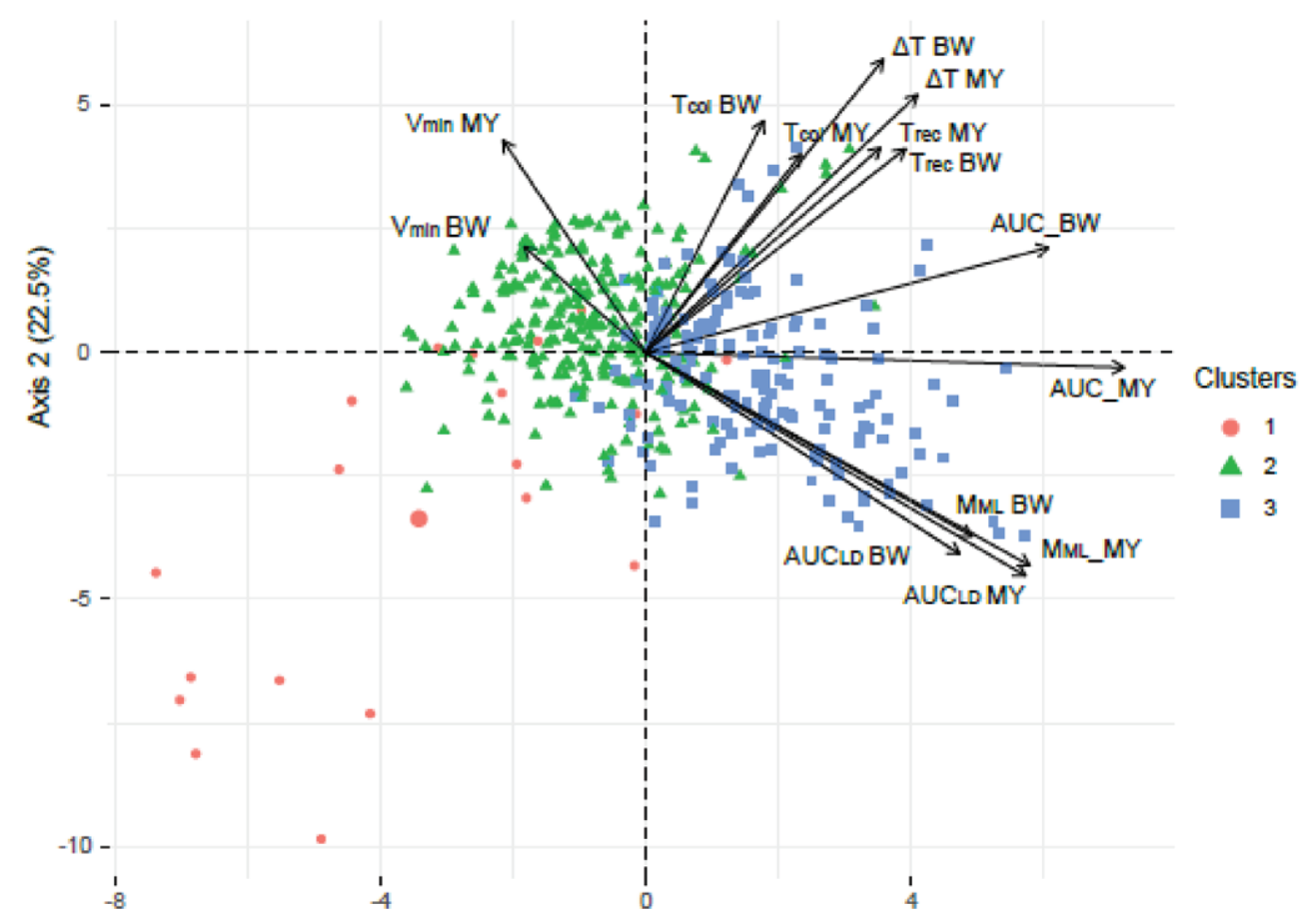

b)

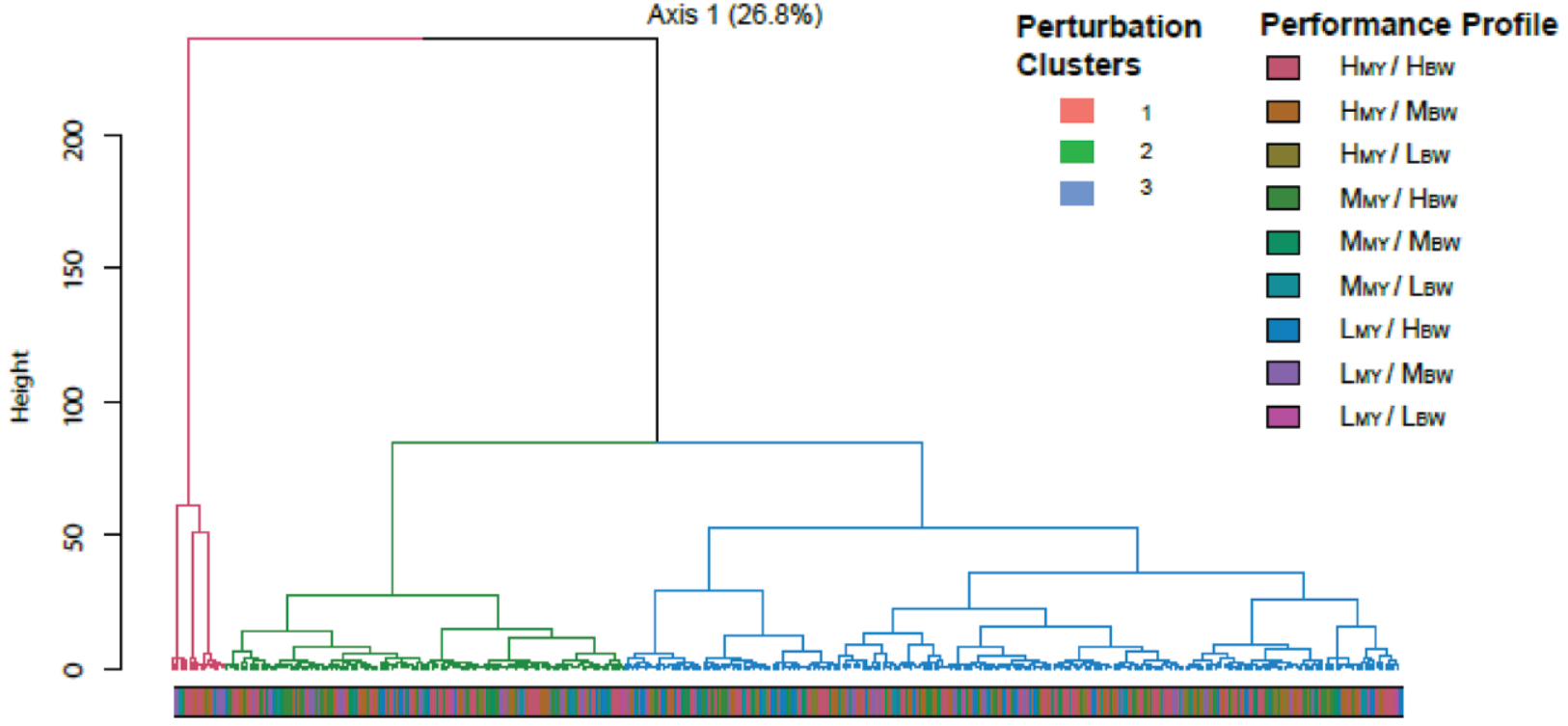

c)

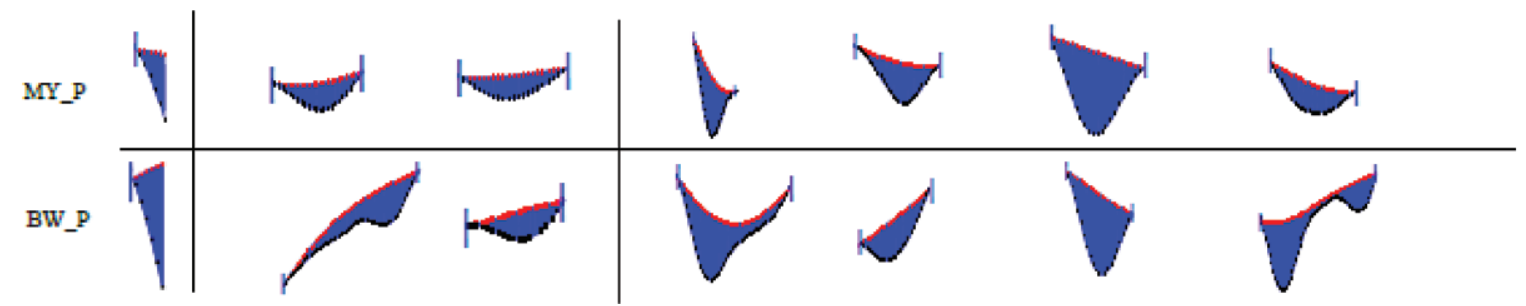

Figure 5. Characterization and classification of concurrent perturbation profiles: (a) biplots of loading and score for the first 2 components of a principal component analysis based on different variables selected to characterize the perturbations; (b) dendrogram highlighting the diversity gradient between the different perturbations and their relation to the performance profiles; and (c) some representative perturbations of each cluster. MY, milk yield; AUC = areas under the curve of the difference between the short-term variation and long-term phenotypic potential performance curves; $\mathrm{AUC}_{\mathrm{LD}}=$ estimation of loss per day $(\mathrm{AUC} / \Delta \mathrm{T}) ; \Delta \mathrm{T}=$ duration of deviation detected; $\mathrm{T}_{\mathrm{col}}=$ collapse phase time by deviation; $\mathrm{T}_{\text {rec }}=$ recovery phase time by deviation; $\mathrm{M}_{\mathrm{ML}}=$ maximum performance loss during deviation; $\mathrm{V}_{\min }=$ minimum value recorded during the deviation. $\mathrm{H}_{\mathrm{MY}}, \mathrm{M}_{\mathrm{MY}}, \mathrm{L}_{\mathrm{MY}}, \mathrm{H}_{\mathrm{BW}}, \mathrm{M}_{\mathrm{BW}}$, and $\mathrm{L}_{\mathrm{BW}}$ represent the cows in the higher, medium, and lower production and BW groups, respectively. 
With this method, it was possible to detect completely synchronous perturbations for the 2 performances (1.2\% of perturbations), and partially synchronous perturbations that start on the same day but with different patterns afterward (17.9\% of perturbations), or perturbations that start at different times but either reach the minimum or the end on different days $(20.2 \%$ and $11.1 \%$ of perturbations, respectively). All types of completely or partially synchronous perturbations are of interest. Given that resilience can be quantified by parameters directly linked to a phase of collapse and recovery from a perturbation, information of additional interest is the extent to which these phases are totally or partially synchronous. The approach of processing 2 performance measures simultaneously gives an additional tool for phenotyping - and perhaps diagnosing - perturbation profiles. Indeed, this information, if coupled with events affecting animal welfare, may help to define the range of different types of responses animals may develop when facing a specific perturbation.

Interestingly, our results showed that the different response phenotypes following perturbations existed in nearly equivalent proportions among the $\mathrm{H}, \mathrm{M}$, and $\mathrm{L}$ production classes, regardless of whether this is assessed on BW or MY trajectories.

We imposed specific filters to eliminate insignificant disturbances and to keep perturbations that seemed to have biological sense. Following these filters, only 425 concurrent perturbations were identified. Given the multifactorial nature of resilience, tools to describe and assess the effect of natural perturbations that occur on commercial farms during the whole lactation period (e.g., disease events, feed quality variation, weather events) could help us obtain a more correct assessment of an animal's performance potential. Understanding how animals adjust their performance under unforeseen circumstances may help herd management (Ollion et al., 2018). Such information is not only important to assess the general phenotypic differences of the sum of the different disturbances during lactation, but can provide the necessary metrics to quantify which are the most resilient individuals. In this context, the methods proposed here could be used on a large scale to phenotype resilience, thereby making possible genetic evaluation and selection for resilience.

Several studies have reported genetic analyses of resilience based on deviations from lactation curves. For example, Elgersma et al. (2018) estimated heritability of traits such as the logarithmic transformation of the variance of daily milk yield, and estimated their genetic correlations with currently evaluated functional traits. However, their approach did not account for the lactation curve. Poppe et al. (2020) also developed resilience indicators by estimating a "potential" lactation curve and then calculating the variance, autocorrelation, and asymmetry of the deviations from individually fitted lactation curves. These authors estimated genetic correlations between these indicators of resilience and characteristics on health, longevity, fertility, metabolism, and production. In contrast, genetic approaches to assess resilience based on BW fluctuation are still relatively uncommon in general (e.g., Berghof et al., $2019 \mathrm{~b}$, in layer chickens), and in particular in dairy cattle.

Other recent studies have tried to predict "resilience" under the assumption that resilient cows are animals that go on to have longer productive lifespans, conceive more easily, and have fewer disease events (Adriaens et al., 2020). Bareille et al. (2003) quantified decrease in feed intake and production losses related to known health disorders. In their case, milk production losses and decrease in feed intake were estimated separately for each health disorder. We suggest that this could be extended using our approach, supplemented by animal

Table 4. Variability of the disturbances according to the chosen variables and the defined clusters by the agglomerative hierarchical clustering

\begin{tabular}{|c|c|c|c|c|c|c|}
\hline \multirow[b]{2}{*}{ Variable $^{1}$} & \multicolumn{2}{|c|}{ Cluster 1 (191) } & \multicolumn{2}{|c|}{ Cluster 2 (215) } & \multicolumn{2}{|c|}{ Cluster 3 (19) } \\
\hline & Milk yield & BW & Milk yield & BW & Milk yield & BW \\
\hline $\mathrm{M}_{\mathrm{AUC}}(\mathrm{kg})$ & 70.54 & 261.77 & 24.95 & 250.83 & 18.48 & 211.53 \\
\hline $\mathrm{M}_{\Delta \mathrm{T}}(\mathrm{d})$ & 34.76 & 34.14 & 28.61 & 33.12 & 16.42 & 25.74 \\
\hline $\mathrm{M}_{\mathrm{Vmin}}(\mathrm{kg})$ & 21.50 & 552.14 & 25.39 & 568.06 & 20.30 & 593.88 \\
\hline $\mathrm{M}_{\mathrm{ML}}(\mathrm{kg})$ & 4.130 & 16.94 & 1.59 & 13.48 & 2.57 & 14.30 \\
\hline $\mathrm{M}_{\mathrm{Tccl}}(\mathrm{d})$ & 16.17 & 15.59 & 14.67 & 16.73 & 12.58 & 19.47 \\
\hline $\mathrm{M}_{\text {Trec }}(\mathrm{d})$ & 18.58 & 18.49 & 13.94 & 16.38 & 3.84 & 6.26 \\
\hline
\end{tabular}




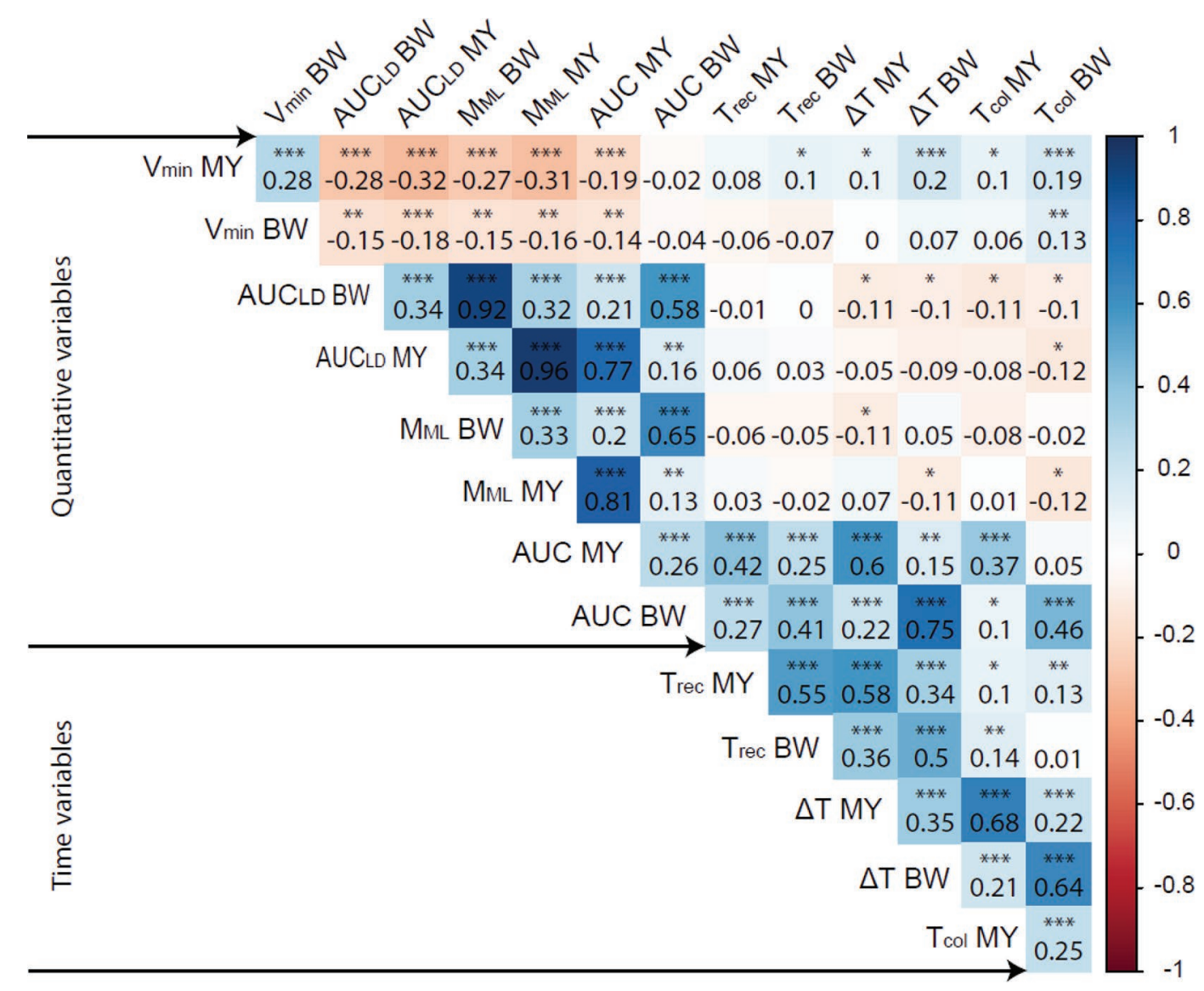

Figure 6. Pearson correlation matrix plot with significance levels between the different variables selected to characterize the perturbations. $\mathrm{MY}=$ milk yield; AUC = areas under the curve of the difference between the short-term variation and long-term phenotypic potential performance curves; $\mathrm{AUC}_{\mathrm{LD}}=$ estimation of loss per day $(\mathrm{AUC} / \Delta \mathrm{T}) ; \Delta \mathrm{T}=$ duration of deviation detected; $\mathrm{T}_{\text {col }}=$ collapse phase time by deviation; $\mathrm{T}_{\text {rec }}=$ recovery phase time by deviation; $\mathrm{M}_{\mathrm{ML}}=$ maximum performance loss during deviation; $\mathrm{V}_{\min }=$ minimum value recorded during the deviation. ${ }^{*} P$-value $\leq 0.05 ; * * P$-value $\leq 0.01 ;{ }^{* * *} P$-value $\leq 0.001$.

health records to improve the precision of resilience phenotyping. Including resilience in selection strategies and farm management may be a key element of effective, elegant, and applicable solutions to find a reasonable compromise between optimal production and animal welfare (www.gentore.eu).

\section{CONCLUSIONS}

This study presents an approach to extract from time-series data different performance classes and different perturbation profiles in MY and BW expressed by dairy cows. It showed that the diversity of shapes of lactation and BW curves is mainly related to the global performance level. Through different penalty coefficients in the fitting procedure, the proposed method also identified perturbations in lactation curves and BW occurring independently or jointly. We suggest that the characteristics of these perturbations can be used as resilience indicators.

\section{ACKNOWLEDGMENTS}

This work was performed in the framework of the DEFFILAIT project (ANR-15-CE20-0014), funded by the French Agence Nationale de la Recherche (ANR, Paris, France) and APIS-GENE (Paris, France). BCELOuest (Locminé, France) and FCEL (Paris, France) are gratefully acknowledged for providing the data from milking robots. The authors have not stated any conflicts of interest.

\section{REFERENCES}

Adriaens, I., N. C. Friggens, W. Ouweltjes, H. Scott, B. Aernouts, and J. Statham. 2020. Productive life span and resilience rank can be predicted from on-farm first-parity sensor time series but not using a common equation across farms. J. Dairy Sci. 103:7155-7171. https://doi.org/10.3168/jds.2019-17826.

Bareille, N., F. Beaudeau, S. Billon, A. Robert, and P. Faverdin. 2003. Effects of health disorders on feed intake and milk production in dairy cows. Livest. Prod. Sci. 83:53-62. https://doi.org/10.1016/ S0301-6226(03)00040-X. 
Ben Abdelkrim, A., L. Puillet, P. Gomes, and O. Martin. 2020. Lactation curve model with explicit representation of perturbations as a phenotyping tool for dairy livestock precision farming. Animal. In press.

Berghof, T. V. L., H. Bovenhuis, and H. A. Mulder. 2019b. Body weight deviations as indicator for resilience in laver chickens. Front. Genet. 10:1216. https://doi.org/10.3389/fgene.2019.01216.

Berghof, T. V. L., M. Poppe, and H. A. Mulder. 2019a. Opportunities to improve resilience in animal breeding programs. Front. Genet. 9:692. https://doi.org/10.3389/fgene.2018.00692.

Caliński, T., and J. Harabasz, ed. 1974. A dendrite method for cluster analysis. Pages 1-27 in Communications in Statistics: Theory and Methods. Bogucki Wydawnictwo Naukowe, Poznań, Poland.

Celeux, G., and G. Govaert. 1992. A classification EM algorithm for clustering and two stochastic versions. Comput. Stat. Data Anal. 14:315-332. https://doi.org/10.1016/0167-9473(92)90042-E.

Codrea, M. C., S. Højsgaard, and N. C. Friggens. 2011. Differential smoothing of time-series measurements to identify disturbances in performance and quantify animal response characteristics: An example using milk yield profiles in dairy cows. J. Anim. Sci. 89:3089-3098. https://doi.org/10.2527/jas.2010-3753.

Colditz, I. G., and B. C. Hine. 2016. Resilience in farm animals: Biology, management, breeding and implications for animal welfare. Anim. Prod. Sci. 56:1961-1983. https://doi.org/10.1071/AN15297.

Elgersma, G. G., G. de Jong, R. van der Linde, and H. A. Mulder. 2018. Fluctuations in milk yield are heritable and can be used as a resilience indicator to breed healthy cows. J. Dairy Sci. 101:12401250. https://doi.org/10.3168/jds.2017-13270.

Everitt, B., S. Landau, and M. Leese. 2001. Cluster Analysis. 4th ed. Arnold, London, UK.

Friggens, N. C., P. Berg, P. Theilgaard, I. R. Korsgaard, K. L. Ingvartsen, P. Løvendahl, and J. Jensen. 2007. Breed and parity effects on energy balance profiles through lactation: Evidence of genetically driven body energy change. J. Dairy Sci. 90:5291-5305. https: //doi.org/10.3168/jds.2007-0173.

Friggens, N. C., C. Duvaux-Ponter, M. P. Etienne, T. Mary-Huard, and P. Schmidely. 2016. Characterizing individual differences in animal responses to a nutritional challenge: Toward improved robustness measures. J. Dairy Sci. 99:2704-2718. https://doi.org/10 $.3168 /$ jds.2015-10162.

Genolini, C., X. Alacoque, M. Sentenac, and C. Arnaud. 2015. kml and kml3d: R packages to cluster longitudinal data. J. Stat. Softw. 65 . https://doi.org/10.18637/jss.v065.i04.

Lebacq, T., P. V. Baret, and D. Stilmant. 2015. Role of input self-sufficiency in the economic and environmental sustainability of specialised dairy farms. Animal 9:544-552. https://doi.org/10.1017/ S1751731114002845.
Martin, O., and A. Ben Abdelkrim. 2019. Detecting perturbations in dairy cows liveweight trajectories. Page 471 in Communications in: Annual Meeting of the European Federation of Animal Science, Ghent, Belgium. European Federation of Animal Science, Ghent, Belgium.

Nguyen Ba, H., J. Van Milgen, and M. Taghipoor. 2019. A procedure to quantify the feed intake response of growing pigs to perturbations. Animal 14:253-260. https://doi.org/10.1017/S1751731119001976.

Ollion, E., F. Blanc, and C. Chassaing. 2018. Des vaches laitières robustes: qu'est-ce les éleveurs en disent? Fourrages (Versailles) 235:219-227.

Poppe, M., R. F. Veerkamp, M. L. van Pelt, and H. A. Mulder. 2020. Exploration of variance, autocorrelation, and skewness of deviations from lactation curves as resilience indicators for breeding. J. Dairy Sci. 103:1667-1684. https://doi.org/10.3168/jds.2019-17290.

Puillet, L., and O. Martin. 2017. A dynamic model as a tool to describe the variability of lifetime body weight trajectories in livestock females. J. Anim. Sci. 95:4846-4856. https://doi.org/10 $.2527 /$ jas2017.1803.

Ramsay, J. O., and B. W. Silverman. 2005. Functional Data Analysis. 1st ed. Springer, New York, NY.

Ramsay, J. O., H. Wickham, S. Graves, and S. Hooker. 2018. Package "fda". R Project. https://cran.r-project.org/web/packages/fda/fda .pdf.

Revilla, M., N. C. Friggens, L. P. Broudiscou, G. Lemonnier, F. Blanc, L. Ravon, M. J. Mercat, Y. Billon, C. Rogel-Gaillard, N. Le Floch, J. Estellé, and R. Muñoz-Tamayo. 2019. Towards the quantitative characterisation of piglets' robustness to weaning: A modelling approach. Animal 13:2536-2546. https://doi.org/10.1017/ S1751731119000843.

Sneath, P. H. A., and R. R. Sokal. 1973. Numerical taxonomy. Page 573 in The Principles and Practice of Numerical Classification. Freeman and Company, San Francisco, CA.

Thorup, V. M., D. Edwards, and N. C. Friggens. 2012. On-farm estimation of energy balance in dairy cows using only frequent body weight measurements and body condition score. J. Dairy Sci 95:1784-1793. https://doi.org/10.3168/jds.2011-4631.

Ward, J. H. Jr. 1963. Hierarchical grouping to optimize an objective function. J. Am. Stat. Assoc. 58:236-244. https://doi.org/10 $.1080 / 01621459.1963 .10500845$.

\section{ORCIDS}

A. Ben Abdelkrim () https://orcid.org/0000-0002-5721-0944

D. Boichard () https://orcid.org/0000-0003-0361-2961 